\title{
Polyphenols and immunity
}

\author{
F. Sánchez de Medina and A. Zarzuelo \\ Department of Pharmacology, School of Pharmacy, University of Granada, Spain
}

Flavonoids are biologically-active polyphenolic compounds with antioxidative, antineoplasic, cardiovascular protective and antiinflammatory properties. Pharmacological therapy is essential in inflammatory bowel disease but has many adverse effects and does not cure the disease. Flavonoids are excellent candidates because of their anti-inflammatory properties and their low toxicity. Several flavonoids have been shown to exert intestinal anti-inflammatory activity in vivo, including ( $\mathrm{mg} / \mathrm{kg}$ ) quercitrin $1-5^{(1)}$, rutin $10-25^{(2)}$, morin $25^{(3,4)}$, hesperidin and diosmin 10-25 $5^{(5)}$. However, the mechanism of action is unclear. Since inflammation is associated by significant oxidative stress, this mechanism may be relevant. Indeed, flavonoid treatment counters colitis-induced glutathione depletion. On the other hand, quercitrin treatment reduces macrophage infiltration in the dextran sulfate sodium colitis model ${ }^{(6)}$. The effects of flavonoids on primary macrophages have been studied and their structure-activity relationship characterized ${ }^{(7)}$. A number of flavonoids inhibit macrophage proliferation (but not cell viability) and some additionally reduce TNF and inducible NO synthase (iNOS) expression, probably interfering with the NF- $\kappa B$ pathway. The structural determinants of activity include the $\mathrm{C}-2=\mathrm{C}-3$ double bond, the catechol group in the $\mathrm{B}$ ring and the 2-position of the $\mathrm{B}$ ring.

Most of these flavonoids are glycosides, which are known to be hydrolysed by bacterial enzymes in the gut. Since luteolin and quercetin are not active in vivo and aglycone flavonoids are absorbed in the small intestine it is likely that glycosides act as prodrugs, releasing the biologically-active aglycone in the lumen and preventing their premature absorption, which has been proven in the case of quercitrin ${ }^{(8)}$. In particular, a faecal homogenate was shown to mediate quercetin release from quercitrin in vitro, and the resulting aglycone retained TNF, iNOS and IL-1 $\beta$ inhibitory activity in murine bone marrow-derived macrophages. This principle probably applies to the other heterosides with known intestinal anti-inflammatory activity.

In conclusion, flavonoids have intestinal anti-inflammatory activity that is associated with macrophage inhibition and antioxidative effects. Further investigation of the mechanistic aspects of flavonoid pharmacological action is underway.

1. Sanchez de Medina F, Galvez J, Romero JA \& Zarzuelo A (1996) J Pharmacol Exp Ther 278, 771-779.

2. Galvez J, Cruz T, Crespo E et al. (1997) Planta Med 63, 409-414.

3. Galvez J, Coelho G, Crespo ME et al. (2001) Aliment Pharmacol Ther 15, 2027-2039.

4. Ocete MA, Galvez J, Crespo ME et al. (1998) Pharmacology 57, 261-270.

5. Crespo ME, Galvez J, Cruz T, Ocete MA \& Zarzuelo A (1999) Planta Med 65, 651-653.

6. Camuesco D, Comalada M, Rodriguez-Cabezas ME et al. (2004) Br J Pharmacol 143, 908-918.

7. Comalada M, Ballester I, Bailon E et al. (2006) Biochem Pharmacol 72, 1010-1021.

8. Comalada M, Camuesco D, Sierra S et al. (2005) Eur J Immunol 35, 584-592. 